\title{
AUTOMATED GENERATION OF FOM MODULES FOR HLA-BASED DISTRIBUTED SIMULATIONS
}

\author{
Paolo Bocciarelli \\ Andrea D'Ambrogio \\ Andrea Giglio \\ Emiliano Paglia \\ Department of Enterprise Engineering \\ University of Rome Tor Vergata \\ Rome, Italy \\ \{paolo.bocciarelli,dambro,andrea.giglio,emiliano.paglia\}@uniroma2.it
}

\begin{abstract}
Distributed Simulation (DS) is widely recognized to provide a valuable support for systems engineering efforts. Various contributions have addressed the adoption of model transformation approaches to enable a more effective DS use, with specific application to the HLA (High Level Architecture) DS standard and the generation of HLA simulation code from SysML system models. This paper extends such contributions by proposing a model transformation approach for the automated generation of the Federation Object Model (FOM), which defines the shared data exchanged during the execution of an HLA DS. The paper specifically addresses the generation of modular FOMs, as introduced by HLA Evolved, the latest version of the HLA standard, and describes the use of the proposed approach as part of a tailored DS development process.
\end{abstract}

Keywords: MDA, model transformation, HLA, DSEEP, FOM, FOM module.

\section{INTRODUCTION}

Distributed simulation (DS) is an effective and natural approach to analyze complex systems, which are very often composed of several subsystems. Despite its acknowledged relevance, the actual adoption of DSbased approaches is often limited in practice, mostly due to the significant effort and skills that are required to properly use DS standards, such as the High Level Architecture (HLA) (IEEE 2010b), and the related implementation technologies.

Methods and tools introduced in the model-driven engineering field, and specifically those based on the Model Driven Architecture (MDA) (OMG 2014), have recently been investigated to mitigate such difficulties and enable an effective DS use in the systems engineering field, as described in (Bocciarelli et al. 2012, Bocciarelli et al. 2015, Kapos et al. 2014, Jain et al. 2015, Topçu et al. 2016). These contributions share a common idea: the DS implementation can be partially obtained by executing a model transformation chain that takes as input an abstract representation of the system under study, typically specified in UML or SysML, and yields as output the skeleton of the DS implementation.

In HLA terms, a DS is referred to as a federation, which results from the interoperation of a set of distributed simulation components, denoted as federates. A DS implementation is not limited to the development of the federates code, but also requires the definition of all shared information that federates agree to exchange at 
execution time. In this respect, a Federation Object Model (FOM) is used to describe the shared information essential to a particular federation (IEEE 2010c).

A major improvement of the latest version of HLA, also known as HLA Evolved (IEEE 2010b), is the introduction of the Modular FOM concept, which allows i) to specify the whole object model across a set of FOM modules, and ii) to extend the FOM during the execution.

This paper focuses on the automated generation of FOM modules and introduces a model-driven approach that extends previous contributions dealing with the generation of federates code (Bocciarelli et al. 2012) or monolithic FOMs (Jain et al. 2015).

Specifically, the paper contribution addresses three different perspectives:

- Process perspective: the DS development process is based on the DSEEP (Distributed Simulation Engineering and Execution Process) standard (IEEE 2010a). Since the adoption of MDA influences the HLA-based DS development, the DSEEP requires to be tailored in order to benefit from the underlying automation layer introduced by model transformations.

- Modeling perspective: in order to drive the automated generation process, the SysML input model has to be annotated with HLA-based concepts. In this respect, this paper proposes a UML profile that is used to carry out the HLA-based annotation of the SysML model.

- Implementation perspective: the implementation of the required model transformation is quite different from the one proposed in previous contributions, because the focus is here shifted from the generation of federate code in a given programming language to the generation of FOM modules, which are serialized in XML.

The reminder of this paper is structured as follows. Section 2 outlines the proposed DSEEP tailoring, Section 3 introduces the UML profile for the annotation of SysML models, while Section 4 describes the proposed model transformation. Section 5 discusses an example application and, finally, Section 6 gives concluding remarks.

\section{DSEEP TAILORING}

The IEEE 1730-2000 DSEEP (Distributed Simulation Engineering and Execution Process) standard (IEEE 2010a) introduces a process model for the development and the execution of a DS exercise.

In order to fully exploit model-driven standards and technologies, the DSEEP needs to be tailored so to include modeling languages and model transformations as an inherent part of the DS development, as shown in Figure 1. As stated in Section 1, under a process perspective, this paper addresses the whole development process, from the definition of federation objectives down to the results analysis. As for the implementation perspective, this paper contribution is instead focused on the automated generation of FOM modules.

In many real world scenarios, the actual simulation implementation may require compliance to a given reference FOM, which is essential to ensure interoperability in a given operational context. Example reference FOMs are the Real-time Platform Reference FOM, for real-time platform-level simulations (SISO 2015), and the Space Reference FOM, for space simulations (Möller et al. 2016). A reference FOM can be used as-is or can be extended to add new simulation concepts that are specific to a particular federation or simulation domain. The proposed approach makes use of a Datatypes Library, which may optionally be included in the SysML model to allow an effective decoupling of the domain model from the concepts and datatypes introduced in the reference FOM. 


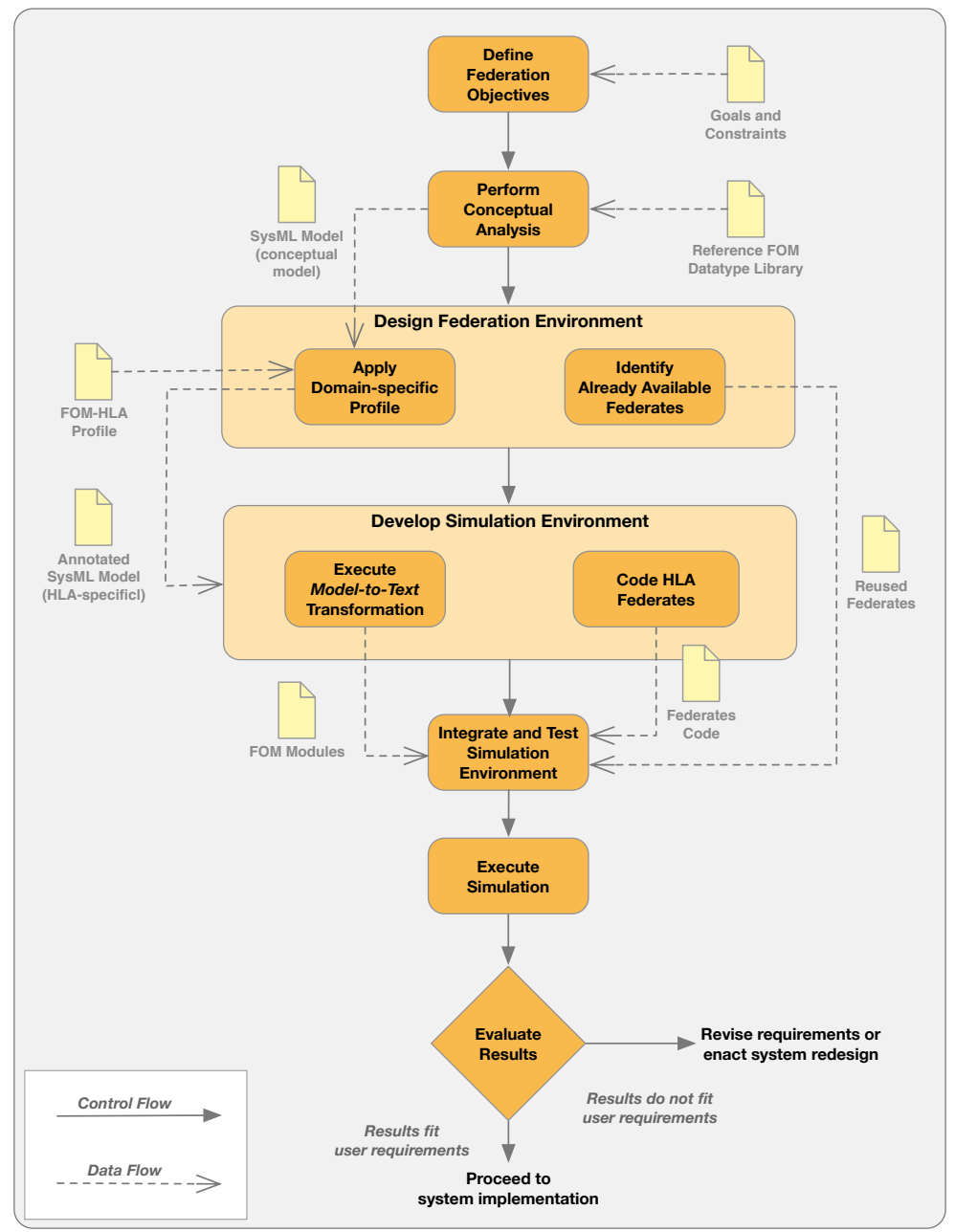

Figure 1: Tailored DSEEP.

The DSEEP tailoring has been designed to ensure compliance to any given FOM reference model. In this respect, in order to keep the method general enough to be used in different domains the tailoring is based on a clear separation of the domain model representation from the concepts and datatypes specified by a reference FOM. To achieve such an objective, the tailored DSEEP exploits two different software artifacts: a Datatypes Library, which provides a SysML representation of datatypes and the object hierarchy specified in the reference FOM, if needed, and a FOM-HLA profile that allows one to annotate the SysML model with FOM and HLA concepts. Different operational contexts may require the use of different reference FOMs (and thus different datatypes libraries), yet the SysML model annotation and the FOM modules generation are carried out using the same FOM-HLA profile and the same model-to-text transformation, respectively.

In details, the process consists of the following activities:

1. At the first step, the simulation objectives and constraints are identified, to clarify what users expect from the DS effort.

2. Then a conceptual analysis is performed to identify the abstract model of the DS that has to be developed. Such a conceptual model is specified in SysML and may optionally include a Datatype Library, if compliance to a given reference FOM is needed. In this respect, the example application 
illustrated in Section 5 shows the use of the tailored DSEEP to generate a DS compliant to the Space Reference FOM (Möller et al. 2016). The resulting SysML model is used as input to the next step.

3. The design federation environment activity is divided in two different sub-activities. First, the SysML model produced at the previous step has to be annotated in order to associate model elements to HLA-related concepts, so to obtain an HLA-specific model. As an example, the annotation allows one to understand which SysML blocks play the role of federates, which block attributes have to be considered as object parameters, etc. The annotation step makes use of the FOM-HLA Profile, which is described in Section 3. The design federation environment step also includes the possible identification of already available federates that are to be included in the federation.

4. Once the annotated SysML model has been defined, along with the possible set of already available federates, the develop federation environment activity is carried out. As in previous step, the federation development consists of two sub-activities: the development of the required FOM modules and the coding of federates. Specifically, the annotated SysML model produced at step 3 is used as input to a model-to-text transformation, which yields as output the set of FOM modules, as described in Section 4. Finally, the implementation of required federates can either be obtained by use of a model-driven approach, as described in (Bocciarelli et al. 2012), or by developing the code from scratch. This step is out of scope and not further detailed.

5. The Integrate and Test Simulation Environment activity is then carried out to put all the pieces together. Existing federates identified at step 3 and new federates developed from scratch at step 4 are integrated to build the concrete DS implementation. A set of tests is also executed to identify and fix possible bugs and to assess the correctness of the simulation behavior.

6. The Execute Simulation activity is then in charge of experimenting the use of the DS to investigate the system behavior and collect results.

7. Finally, the DS results are analyzed to investigate the behavior of the simulated system in its operational environment, thus providing systems engineers with relevant information to plan possible re-design and implementation tasks.

The next two sections describe the FOM-HLA profile and the model-to-text transformation that yields as output the set of FOM modules, respectively.

\section{FOM-HLA PROFILE}

As stated in Section 1, previous contributions have proposed MDA-based approaches to ease the development of federates code. In this respect, two UML profiles have been introduced, namely the SysML4HLA and the HLA profiles, to enable the annotation of SysML models with HLA-related concepts. Specifically, while the main objective of the SysML4HLA profile is the identification of those SysML elements playing an active role in the HLA simulation (e.g., SysML blocks acting as object classes or attributes which have to be considered object parameters, etc.) and it is used to generate a UML model of the simulation, the HLA profile focuses on the representation of HLA-based implementation details (e.g., publish/subscribe relationships, time management, etc.).

This paper extends such previous contributions and introduces the FOM-HLA profile, which is used to annotate the SysML model with what is needed to drive the execution of the model-to-text transformation that generates the set of FOM modules.

The FOM-HLA Profile consists of a set of stereotypes, which are defined as extensions of standard UML metaclasses, and is organized in two different packages: the OMTKernel package, which contains stereotypes used to annotate the model, and the HLADatatypes package, which provides the data types of the attributes used to specify the stereotypes included in the OMTKernel package. Both packages refer to the 

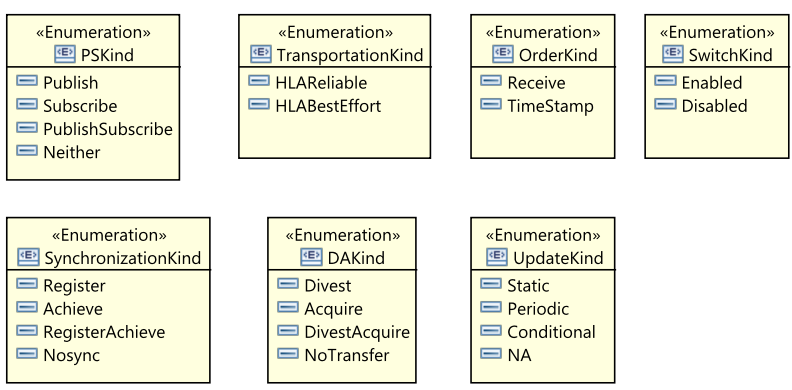

Figure 2: FOM-HLA SysML Profile: Datatype Package.

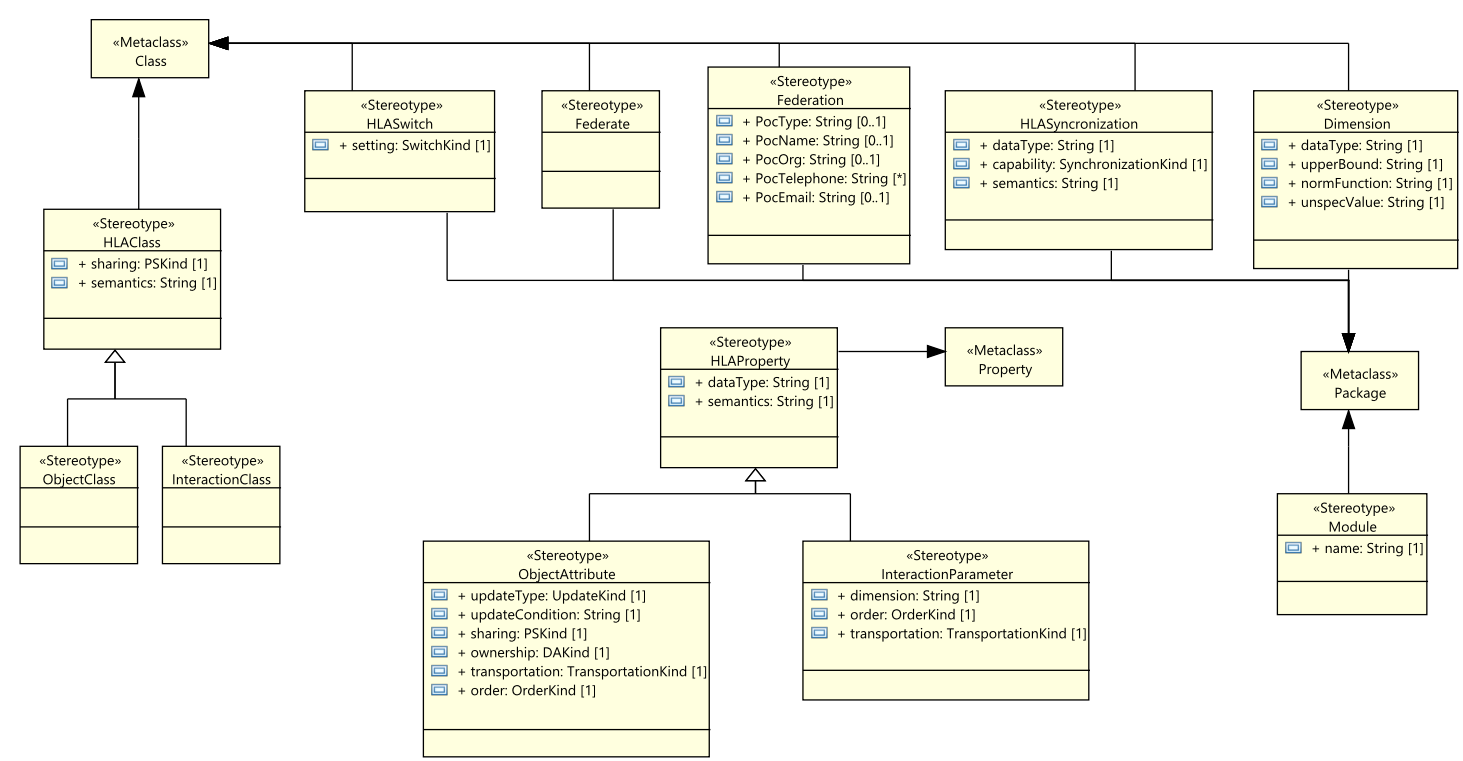

Figure 3: FOM-HLA SysML Profile: OMTKernel Package.

HLA Object Model Template (OMT), the standard that defines the format of HLA FOMs. The reader if referred to (IEEE 2010c) for a detailed description of OMT types and objects.

Figure 2 illustrates the HLADatatypes package, which provides a set of enumerated types according to the OMT types, while Figure 3 illustrates the OMTKernel package, which provides the set of following stereotypes extending standard UML metaclasses:

- Module: a package element containing model elements which must be mapped to a specific FOM module. It is specified by the tagged value:

- Name: the FOM module name.

- Federate: a federate within a federation.

- ObjectClass: an object class.

- InteractionClass: an interaction class.

- Dimension: a specific dimension for an attribute of an Objectclass or an Interactionclass, specified by tagged values:

- DataType: the datatype for the federate view of the dimension;

- UpperBound: the upper bound that the federation requirements allow; 
- UnspecifiedValue: a default value used when the dimension value is unspecified.

- NormFunction: the normalization function.

- HLAProperty: the abstract concept of properties that is specialized by ObjectAttribute and InteractionParameter stereotypes. It is specified by tagged values:

- DataType: datatype of attributes or parameters.

- Semantics: semantics of attributes or parameters.

- ObjectAttribute: an object class attribute, specified by tagged values:

- UpdateType: the policy for updating an attribute;

- UpdateCondition: the condition that must be met for updating the attribute;

- Sharing: the publish/subscribe capabilities of a federate with respect to an attribute;

- Ownership: the ownership of an instance of a class attribute (released or acquired);

- Transportation: the transportation type to be used;

- Order: the order of delivery.

- InteractionParameter: an interaction class attribute, specified by tagged values:

- Dimension: the association of a class attribute with a set of dimensions;

- Transportation: the transportation type to be used;

- OrderKind: the order of delivery.

- HLAClass: abstraction of object class and interaction class, specified by tagged values:

- Sharing: the information on publication and subscription capabilities;

- Semantics: the semantics of the HLA class.

- HLASynchronization: the synchronization points used to synchronize federates during the federation execution, specified byy tagged values:

- Datatype: the datatype used for the user-supplied tag (when applicable);

- Capability: the capability of federates with respect to each synchronization point;

- Semantics: the semantics of the synchronization point.

- HLASwitch: the initial settings of several run-time infrastructure switches, specified by the tagged value:

- Setting: the initial setting for each switch.

\section{TRANSFORMATION SPECIFICATION}

The generation of the required FOM modules is carried out by use of a model-to-text transformation implemented in Acceleo, the model-driven Eclipse plugin for code generation (Eclipse Foundation 2015). The model transformation takes as input the SysML model of the system, annotated with stereotypes provided by the FOM-HLA profile, and yields as output the required set of FOM modules. The next three subsections illustrate the mapping rationale and the handling of subclassing and modularity, respectively.

\subsection{Mapping Overview}

As stated in Section 2, the proposed DSEEP tailoring introduces a model-to-text transformation to generate the required set of FOM modules. The SysML model representing the system to be simulated exploits two different artifacts to include what is needed to drive such a transformation: the HLA-FOM profile, which allows one to specify HLA-based annotations, and, optionally, a library specifying the datatypes and the objects hierarchy which have to be used in order to make the simulation compliant to a given reference FOM. Table 1 identifies the source of (where to find) the various input elements that the transformation uses to generate the corresponding FOM module elements. 
Table 1: Data source for FOM module elements.

\begin{tabular}{|c|c|}
\hline Element & Source \\
\hline \multicolumn{2}{|l|}{ Identification Table } \\
\hline Name & SysML model \\
\hline Type & SysML model \\
\hline Modification date & date of transformation execution \\
\hline POC & Profile \\
\hline \multicolumn{2}{|l|}{ Object Classes } \\
\hline Object Class name & SysML model (if stereotyped) \\
\hline Object Class semantics & Profile \\
\hline Class Hierarchy & SysML model + SysML library \\
\hline \multicolumn{2}{|l|}{ Object Attributes } \\
\hline Attribute name & SysML model (if stereotyped) \\
\hline Attribute datatype & SysMl model + SysML library \\
\hline Attribute semantics & Profile \\
\hline Attribute characterization ${ }^{1}$ & Profile \\
\hline \multicolumn{2}{|l|}{ Interaction Classes } \\
\hline Interaction Class name & SysML model (if stereotyped) \\
\hline Interaction Class semantics & Profile \\
\hline Class Hierarchy & SysML model + SysML library \\
\hline \multicolumn{2}{|l|}{ Interaction Parameters } \\
\hline Parameter name & SysML model (if stereotyped) \\
\hline Parameter datatype & SysMl model + SysML library \\
\hline Parameter semantics & Profile \\
\hline Parameter characterization ${ }^{1}$ & Profile \\
\hline $\begin{array}{ll}\text { Datatypes } \\
\end{array}$ & \\
\hline Name, type, hierarchy, semantics & SysML library \\
\hline $\begin{array}{ll}\text { Switches } \\
\end{array}$ & \\
\hline All attributes & Profile \\
\hline $\begin{array}{r}\text { Synchronization points } \\
\text { All attributes }\end{array}$ & Profile \\
\hline
\end{tabular}

Table 1 outlines the rationale of the model transformation, but does not clarify how to deal with two important transformation issues, i.e., the partitioning of the FOM into different modules and the compliance to a reference FOM. Such issues are discussed in Section 4.2 and Section 4.3, respectively.

\subsection{Handling of Modularity}

One of the main requirements that the model transformation has to satisfy is the handling of modularity. From a general perspective, the SysML model provides a representation of the whole simulation system. According to the HLA Evolved standard, the FOM is not necessarily to be described in a monolithic document, but can be specified throughout a set of modules, each providing a subset of the whole model. In

\footnotetext{
${ }^{1}$ According to the HLA standard, the attribute/parameter characterization briefly denotes the specification of Update type, Condition, Ownership, Sharing, Dimension, Transportation and Order.
} 

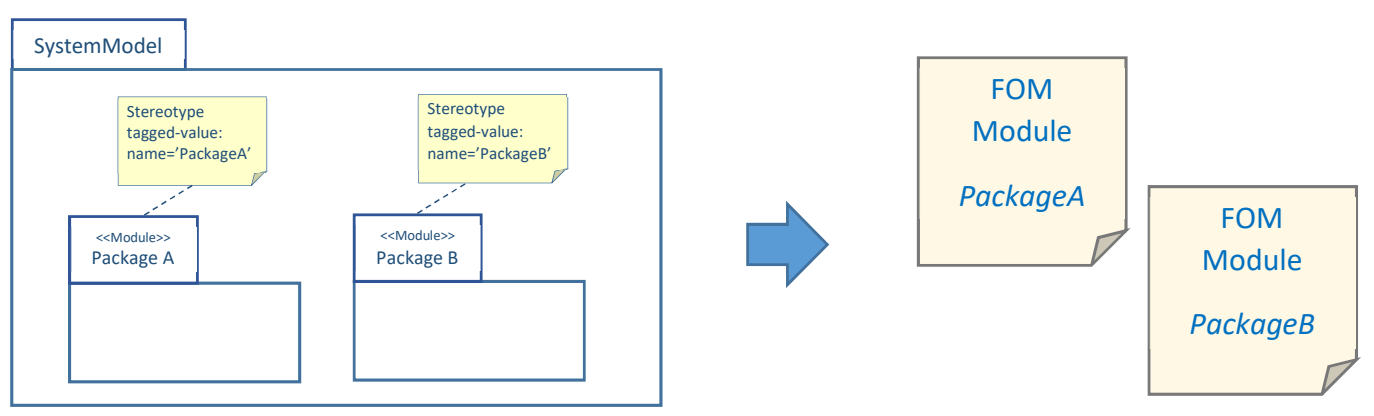

Figure 4: Handling of modularity.

this respect, the proposed method enables the partitioning of the FOM into a set of modules by use of the following strategy:

- the system model is partitioned in different packages, so that model elements that have to be mapped to the same FOM module are contained in the same package;

- each package is annotated with the «Module» stereotype provided by the FOM-HLA Profile, with the tag Name used to specify the related module name;

- the model transformation processes one package at a time, in order to yield as output the required FOM modules, as shown in Figure 4.

\subsection{Handling of Subclassing}

An important objective of the proposed approach is to specify a model transformation that is general enough to be used in different application domains and operational contexts. In other words, the transformation must not assume the availability of a specific reference FOM and must not hard-code any predefined FOM structure. To achieve such an objective, the proposed approach makes use of an external SysML library, which defines the datatypes and the objects hierarchy as specified by the optional reference FOM.

In this respect, the availability of the external (and optional) library allows systems engineers to make the model compliant to the reference FOM by specifying that i) a model element stereotyped as «ObjectClass» or «InteractionClass» is a subclass of a given library element and, ii) an attribute of the source model stereotyped as «ObjectAttribute» or «InteractionParameter» must be typed by one of the datatypes provided by the library.

This design choice requires the model-to-text transformation to effectively handle inheritance relationships by considering two different types of inheritance relationship. To better explain such an idea, let us consider the example shown in Figure 5, where a SysML model uses an external Datatype Library that refers to the Space Reference FOM.

The left part of the figure shows the model given as input to the transformation engine. The Datatype Library provides an implementation of the entity hierarchy specified in the Space Reference FOM and the implementation of the several related datatypes, as well.

The first type of inheritance relationship that the transformation has to address is the one existing in the actual annotated SysML model: as shown in the figure, BlockC is a subclass of BlockB which in turn is a subclass of BlockA. The stereotypes «ObjectClass» and «ObjectAttribute» are used to specify which 


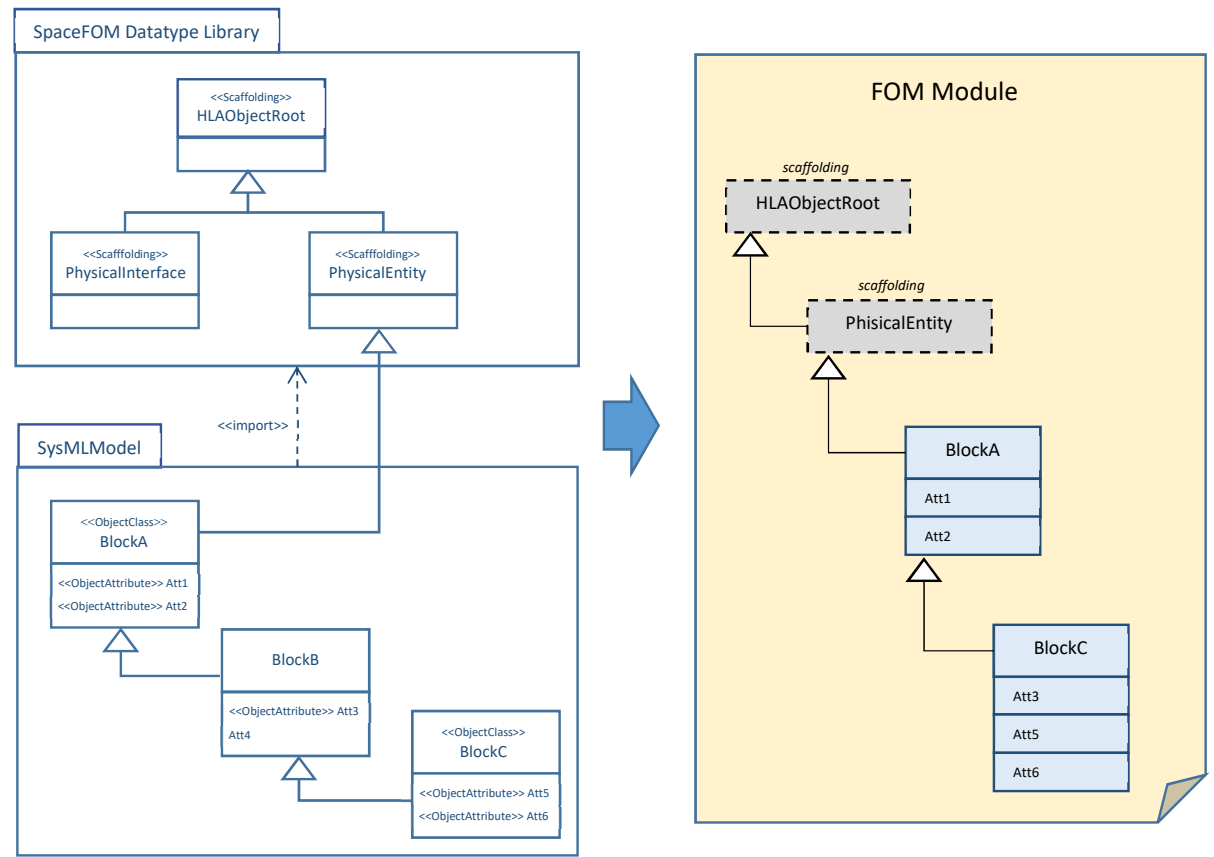

Figure 5: Handling of subclassing.

elements play the relevant role in the HLA federation. It should be underlined that it is not necessary that all the elements in the model have a counterpart in the FOM.

The second type of inheritance relationship that the transformation has to address is the one between model elements and the elements of the imported library, when available, e.g., the subclassing with respect to the entities provided by the reference FOM. In the example shown in Figure5, BlockA plays the role of an object class that inherits from the PhysicalEntity element, so to properly manage the scaffolding mechanism that makes the resulting FOM module compliant to the Space Reference FOM.

\section{EXAMPLE APPLICATION}

Let us consider the design and implementation of the Control Unit $(C U)$ software module, which constitutes the core of the Orbiter Space System. As the CU is a mission critical system, a simulation-based analysis must be carried out to assess the correct behavior of the CU, before its actual implementation. Let us suppose that the Space Federation for simulating a space system is also available, so that the simulation of the Orbiter must be carried out by implementing an Orbiter Federate, part of the Space Federation. Moreover, in order to be compliant with the Space Federation, the orbiter federate must conform to the Space Reference FOM (SpaceFOM). Finally, it is also assumed that the SpaceFOM Datatype Library has been made available, to enable the specification of federates compliant to the Space Federation. Figure 6 summarizes the relationship among the several software items that make the Orbiter SysML model:

- SpaceFOM Datatype Library: this library contains a SysML implementation of the several datatypes specified in the Space Reference FOM, e.g., velocity, velocity vector, position vector, etc. The library also provides the implementation of entities hierarchy, e.g., the object class DinamicEntity, as subclass of PhysicalEntity, which in turn is subclass of HLAobjectRoot. 


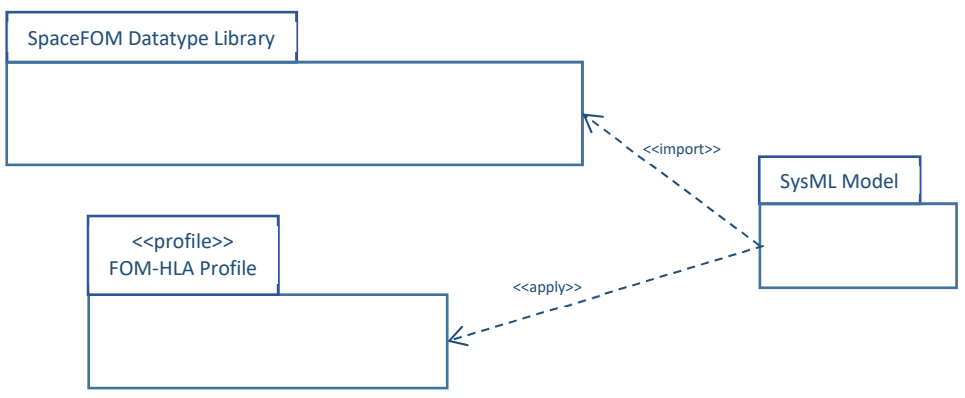

Figure 6: Relationship among simulation system model, FOM-HLA profile and Datatype library.

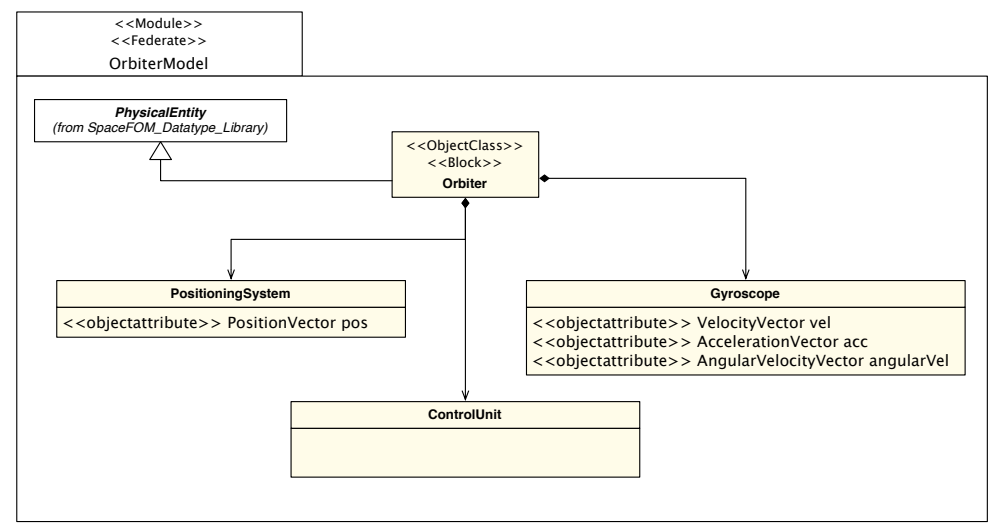

Figure 7: Annotated Orbiter Conceptual Model.

- FOM-HLA Profile: the profile includes a set of stereotypes to specify the required HLA-related concepts used to generate the FOM modules, e.g., «ObjectClass» to annotate SysML model blocks which play the role of object classes in the federation, «SynchronizationPoint» to specify additional synchronization points, etc.

As state in Section 1, the development of simulation code has been discussed in our past work and it is out of the scope of this paper. Indeed, this example aims to show how the proposed method can be effectively adopted to generate a FOM module compliant to a given reference FOM.

According to the tailored DSEEP shown in Figure 1, once the simulation objective has been clearly identified, the conceptual analysis is executed to specify an abstract model of the Orbiter system. Indeed, the output of this step is the orbiter conceptual model, which makes use of the SpaceFOM Datatype Library.

At the next step (Design Federation Environment), the model is annotated with stereotypes provided by the HLA-FOM profile. The resulting SysML model is shown in Figure 7.

The so obtained conceptual model is given as input to the model-to-text Acceleo transformation that is executed to generate the required FOM modules. According to the annotation specified in Figure 7, the transformation output only includes the Orbiter_FOM module.

A fragment of the Orbiter_FOM module is shown in Listing 2, while Listing 1 includes a fragment of the Space Reference FOM. It is worth noting how object classes and interaction classes of the Orbiter_FOM conform to the hierarchy specified by the Space Reference FOM via the scaffolding declaration mechanism. 
Listing 1: Fragment of Space Reference FOM.

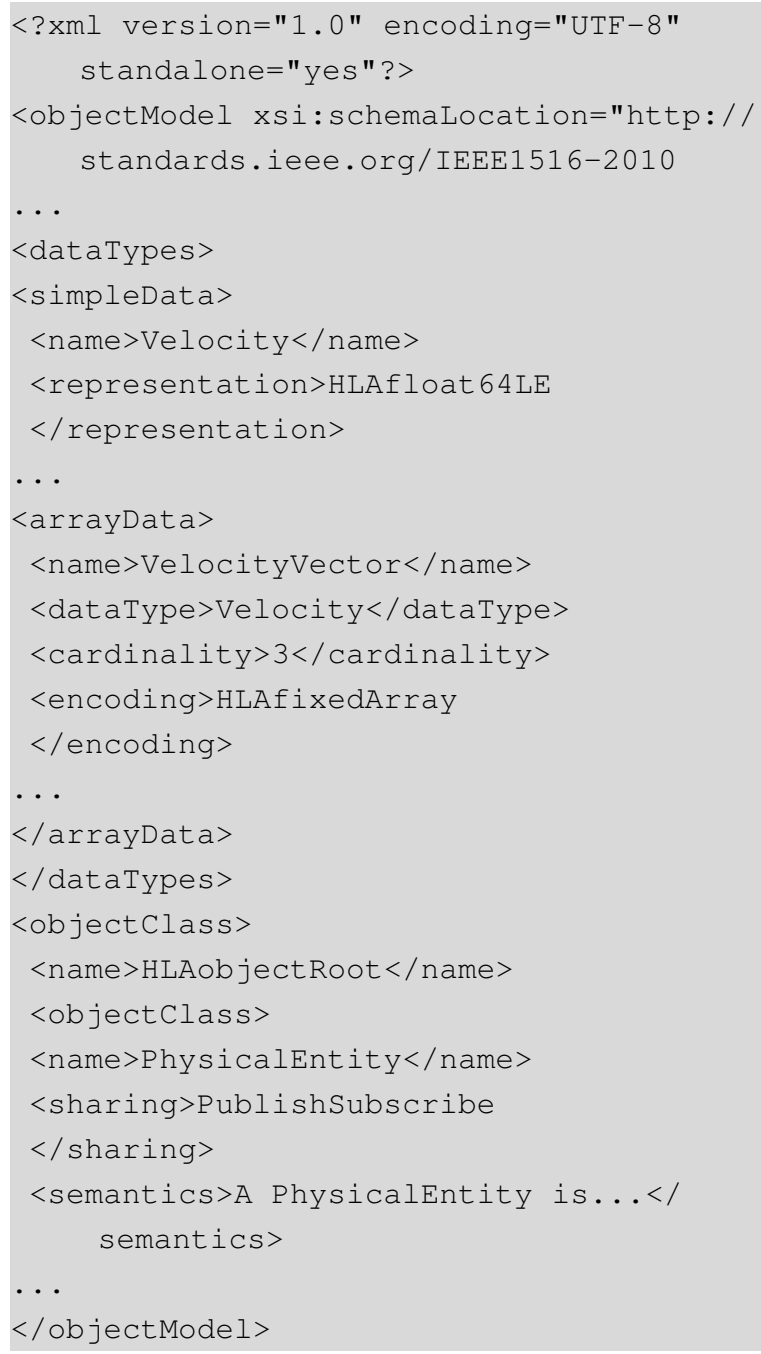

Listing 2: Fragment of Orbiter_FOM Module.

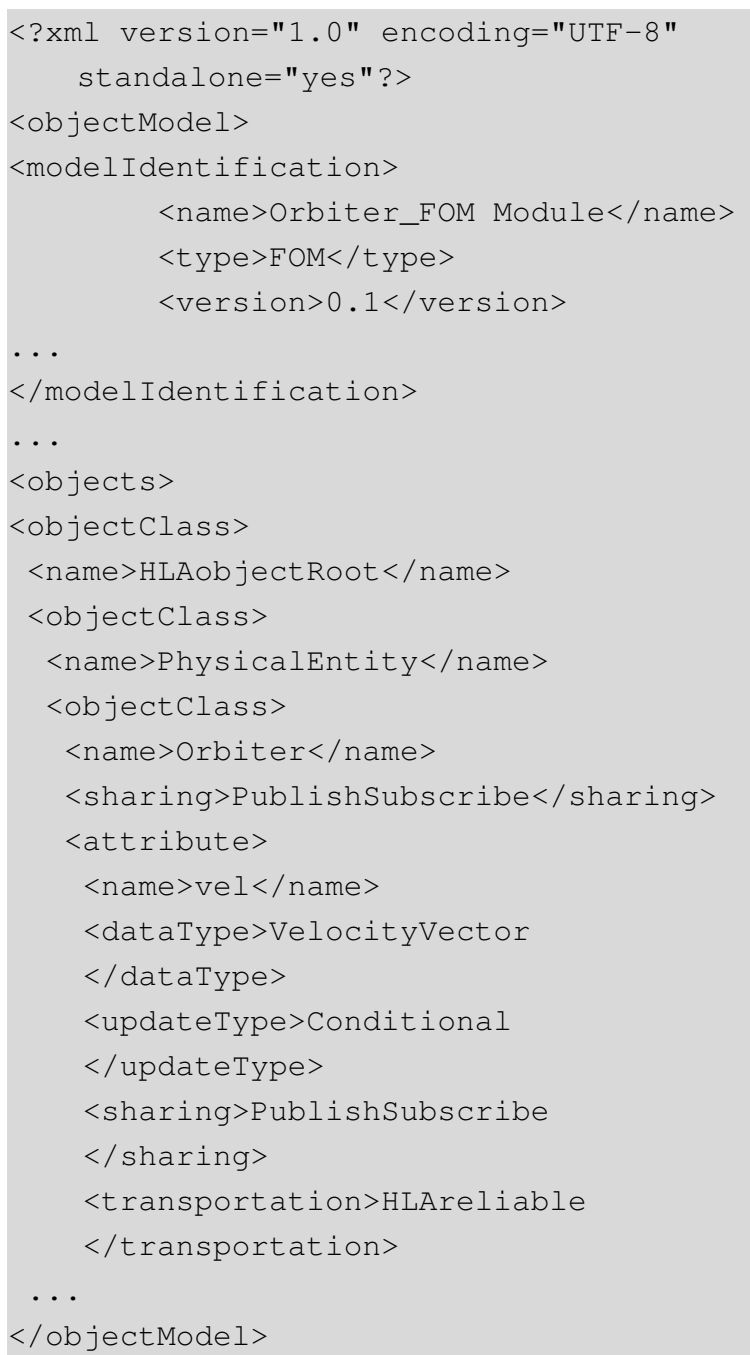

$</$ objectModel $>$

\section{CONCLUSIONS}

The paper has introduced a model transformation approach for the automated generation of the Federation Object Model (FOM) in HLA-based distributed simulations. The proposed approach is intended to be used as part of a tailored DS development process, which is founded on the use of modeling techniques and model transformation approaches to enable a more effective DS adoption for model-based systems engineering. The proposed approach has specifically addressed the generation of modular FOMs, as introduced by HLA Evolved, the latest version of the HLA standard, and can be used to generate FOMs that provide compliance to standardized reference FOMs.

\section{REFERENCES}

Bocciarelli, P., A. D'Ambrogio, and G. Fabiani. 2012. "A Model-driven Approach to Build HLA-based Distributed Simulations from SysML Models". In Proceedings of the 2nd International Conference on Simulation and Modeling Methodologies, Technologies and Applications, SIMULTECH'12, pp. 49-60. 
Bocciarelli, P., A. D'Ambrogio, A. Falcone, A. Garro, and A. Giglio. 2015. "A model-driven approach to enable the distributed simulation of complex systems". In Proceedings of the 6th International Conference on Complex Systems Design and Management, CSDM. Paris, France.

Bocciarelli, P., A. Pieroni, D. Gianni, and A. D'Ambrogio. 2012. "A model-driven method for building distributed simulation systems from business process models". In Proceeding of the 2012 Winter Simulation Conference, WSC 2012, pp. 227-238.

Eclipse Foundation 2015. "Acceleo". Website: https://eclipse.org/acceleo/.

IEEE 2010a. 1730-2010 Distributed Simulation Engineering and Execution Process (DSEEP).

IEEE 2010b. IEEE 1516 - Standard for Modeling and Simulation High Level Architecture - Framework and Rules.

IEEE 2010c. IEEE 1516.2- Standard for Modeling and Simulation High Level Architecture - Object Model Template (OMT) Specification.

Jain, A., R. Fujimoto, J. Crittenden, M. Liu, J. Kim, and Z. Lu. 2015. "Towards automating the development of federated distributed simulations for modeling sustainable urban infrastructures". In Winter Simulation Conference (WSC), 2015, pp. 2668-2679. IEEE.

Kapos, G.-D., V. Dalakas, A. Tsadimas, M. Nikolaidou, and D. Anagnostopoulos. 2014. "Model-based system engineering using SysML: Deriving executable simulation models with QVT". In Systems Conference (SysCon), 2014 8th Annual IEEE, pp. 531-538. IEEE.

Möller, B., E. Z. Crues, D. Dexter, A. Garro, A. Skuratovskiy, and A. Vankov. 2016. "A First Look at the Upcoming SISO Space Reference FOM”. In SISO 2016 Simulation Innovation Workshop (SIW).

OMG 2014. MDA Guide, version 2.0.

SISO 2015. Standard for Real-time Platform Reference Federation Object Model version 2.0. Simulation Interoperability Standards Organization - SISO-STD-001.1-2015.

Topçu, O., U. Durak, H. Oğuztüzün, and L. Yilmaz. 2016. Distributed Simulation: A Model Driven Engineering Approach. Springer.

\section{AUTHOR BIOGRAPHIES}

PAOLO BOCCIARELLI is a postdoc researcher at the Department of Enterprise Engineering of the University of Roma Tor Vergata (Italy). His research interests include software and systems engineering, business process management, model-driven development and distributed simulation. His email address is paolo.bocciarelli@uniroma2.it.

ANDREA D'AMBROGIO is associate professor of systems and software engineering at the Department of Enterprise Engineering of the University of Roma Tor Vergata (Italy). His research interests are in the areas of system performance and dependability engineering, model-driven systems and software engineering, business process management, and distributed simulation. His email address is dambro@uniroma2.it.

ANDREA GIGLIO is a postdoc researcher at the Department of Enterprise Engineering of the University of Roma Tor Vergata (Italy). His research interests include model-driven system and software engineering and business process management. His email address is andrea.giglio@uniroma2.it.

EMILIANO PAGLIA is a postdoc researcher at the Department of Enterprise Engineering of the University of Roma Tor Vergata (Italy). His research interests include model-driven engineering and business process modeling and analysis. His email address is emiliano.paglia@uniroma2.it. 